\title{
Fixed Points of Meromorphic Solutions for Some Difference Equations
}

\author{
Zong-Xuan Chen ${ }^{1}$ and Kwang Ho Shon ${ }^{2}$ \\ ${ }^{1}$ School of Mathematical Sciences, South China Normal University, Guangzhou 510631, China \\ ${ }^{2}$ Department of Mathematics, College of Natural Sciences, Pusan National University, Pusan 609-735, Republic of Korea \\ Correspondence should be addressed to Kwang Ho Shon; khshon@pusan.ac.kr
}

Received 1 December 2012; Accepted 17 April 2013

Academic Editor: Patricia J. Y. Wong

Copyright (c) 2013 Z.-X. Chen and K. H. Shon. This is an open access article distributed under the Creative Commons Attribution License, which permits unrestricted use, distribution, and reproduction in any medium, provided the original work is properly cited.

We investigate fixed points of meromorphic solutions $y(z)$ for the Pielou logistic equation and obtain some estimates of exponents of convergence of fixed points of $y(z)$ and its shifts $y(z+n)$, differences $\Delta y(z)=y(z+1)-y(z)$, and divided differences $\Delta y(z) / y(z)$.

\section{Introduction and Results}

In this paper, we assume the reader is familiar with basic notions of Nevanlinna's value distribution theory (see [1-3]). In addition, we use the notation $\sigma(f)$ to denote the order of growth of a meromorphic function $f$ and $\lambda(f)$ and $\lambda(1 / f)$ to denote, respectively, the exponents of convergence of zeros and poles of $f$. We also use the notation $\tau(f)$ to denote the exponent of convergence of fixed points of $f$ that is defined as

$$
\tau(f)=\varlimsup_{r \rightarrow \infty} \frac{\log N(r, 1 /(f(z)-z))}{\log r} .
$$

Recently, a number of papers (including [4-17]) focus on complex difference equations and difference analogues of Nevanlinna's theory.

The Pielou logistic equation

$$
y(z+1)=\frac{R(z) y(z)}{Q(z)+P(z) y(z)},
$$

where $P(z), Q(z)$, and $R(z)$ are nonzero polynomials, is an important difference equation, because it is obtained by transform from the well-known Verhulst Pearl equation (see [18, page 99])

$$
x^{\prime}(t)=x(t)[a-b x(t)] \quad(a, b>0),
$$

which is the most popular continuous model of growth of a population.

Chen [7] obtained the following theorem.

Theorem A. Let $P(z), Q(z)$, and $R(z)$ be polynomials with $P(z) Q(z) R(z) \neq \equiv 0$ and let $y(z)$ be a finite order transcendental meromorphic solution of (2). Then

$$
\lambda\left(\frac{1}{y}\right)=\sigma(y) \geq 1 .
$$

Example 1. The function $y(z)=z 2^{z} /\left(2^{z}-1\right)$ satisfies the Pielou logistic equation

$$
y(z+1)=\frac{2(z+1) y(z)}{z+y(z)},
$$

where $y(z)$ satisfies

$$
(y)=0, \quad \lambda\left(\frac{1}{y}\right)=\sigma(y)=1 .
$$

This example shows that the result of Theorem A is sharp.

One of the main purposes in this paper is to study fixed points of meromorphic solutions of the Pielou logistic equation (2).

The problem of fixed points of meromorphic functions is an important one in the theory of meromorphic functions. 
Many papers and books (including [18-20]) investigate fixed points of meromorphic functions.

Now we consider fixed points of meromorphic functions and their shifts, differences, and divided differences. We see that there are many examples to show that either $f(z)$ may have no fixed point, for example, $f_{1}(z)=e^{z}+z$ or the shift $f(z+c)$ of $f(z)$, or the difference $\Delta_{c} f(z)=f(z+c)-f(z)$ of $f(z)$ may have only finitely many fixed points; for example, for the function $f_{2}(z)=e^{z}+z-1$, its shift $f_{2}(z+1)=e e^{z}+z$, and its difference $\Delta_{2 \pi i} f_{2}(z)=f_{2}(z+2 \pi i)-f_{2}(z)=2 \pi i$ have only finitely many fixed points. Even if for a meromorphic function of small growth, Chen and Shon show that there exists a meromorphic function $f_{0}$ such that $\sigma\left(f_{0}\right)<1$ and $\Delta_{c} f_{0}(z)=f_{0}(z+c)-f_{0}(z)$ has only finitely many fixed points (see Theorem 6 of [9]).

A divided difference $(f(z+c)-f(z)) / f(z)$ may also have only finitely many fixed points; for example, the function $f(z)=z e^{z}$ satisfies that its divided difference $(f(z+1)-$ $f(z)) / f(z)=((z+1) e-z) / z$ has only finitely many fixed points. Chen and Shon obtained Theorem B.

Theorem B (see [9]). Let $c \in \mathbb{C} \backslash\{0\}$ be a constant and let $f$ be a transcendental meromorphic function of order of growth $\sigma(f)=\sigma<1$ or of the form $f(z)=h(z) e^{a z}$, where $a \neq 0$ is a constant and $h(z)$ is a transcendental meromorphic function with $\sigma(h)<1$. Suppose that $p(z)$ is a nonconstant polynomial. Then

$$
G(z)=\frac{f(z+c)-f(z)}{f(z)}-p(z)
$$

has infinitely many zeros.

From Theorem B, we easily see that under conditions of Theorem $\mathrm{B}$, the divided difference $G_{1}(z)=(f(z+c)-$ $f(z)) / f(z)$ has infinitely many fixed points. The previous example $f(z)=z e^{z}$ shows that result of Theorem B is sharp.

However, we discover that the properties on fixed points of meromorphic solutions of (2) are very good. We prove the following theorem.

Theorem 2. Let $P(z), Q(z)$, and $R(z)$ be nonzero polynomials such that

$$
\operatorname{deg} P(z) \geq \max \{\operatorname{deg} R(z), \operatorname{deg} Q(z)\}, \quad \operatorname{deg} P(z) \geq 1 .
$$

Set $\Delta y(z)=y(z+1)-y(z)$. Then every finite order transcendental meromorphic solution $y(z)$ of (2) satisfies the following:

(i) $\tau(y(z+n))=\sigma(y(z)) \geq 1(n=0,1, \ldots)$;

(ii) if $R(z)-(z+1) Q(z) \not \equiv 0$, then $\tau(\Delta y(z) / y(z))=$ $\sigma(y(z))$;

(iii) if there is a polynomial $h(z)$ satisfying

$$
(-R(z)+Q(z)+z P(z))^{2}-4 z P(z) Q(z)=h(z)^{2},
$$

then $\tau(\Delta y(z))=\sigma(y(z))$.
Remark 3. Generally, $\tau(f(z)) \neq \tau(f(z+c))$ for a meromorphic function $f(z)$ of finite order. For example, the function $f_{1}(z)=e^{z}+z$ satisfies

$$
\tau\left(f_{1}(z)\right)=0 \neq \tau\left(f_{1}(z+1)\right)=1 .
$$

\section{Proof of Theorem 2}

We need the following lemmas for the proof of Theorem 2.

Lemma 4 (see $[12,17])$. Let $w(z)$ be a nonconstant finite order meromorphic solution of

$$
P(z, w)=0
$$

where $P(z, w)$ is a difference polynomial in $w(z)$. If $P(z, a) \not \equiv 0$ for a meromorphic function $a(z)$ satisfying $T(r, a)=S(r, w)$, then

$$
m\left(r, \frac{1}{w-a}\right)=S(r, w)
$$

holds for all $r$ outside of a possible exceptional set with finite logarithmic measure.

Remark 5. Using the same method as in the proof of Lemma 4 (see [12]), we can prove that in Lemma 4, if all coefficients $b_{\lambda}(z)$ of $P(z, w)$ satisfy $\sigma\left(b_{\lambda}(z)\right)=\sigma_{1}<\sigma(w(z))=$ $\sigma$ and if $P(z, a) \not \equiv 0$ for a meromorphic function $a(z)$ satisfying $T(r, a)=S(r, w)$, then for a given $\varepsilon\left(0<\varepsilon<\sigma-\sigma_{1}\right)$,

$$
m\left(r, \frac{1}{w(z)-a(z)}\right)=S(r, w(z))+O\left(r^{\sigma_{1}+\varepsilon}\right)
$$

holds for all $r$ outside of a possible exceptional set with finite logarithmic measure.

Lemma 6. Suppose that $R(z), Q(z)$, and $P(z)$ satisfy the condition (8) in Theorem 2 and that $y(z)$ is a nonconstant meromorphic function. Then

$$
\begin{gathered}
f_{1}(z)=(R(z)-z P(z)) y(z)-z Q(z), \\
f_{2}(z)=P(z) y(z)+Q(z)
\end{gathered}
$$

have at most finitely many common zeros.

Proof. Suppose that $z_{0}$ is a common zero of $f_{1}(z)$ and $f_{2}(z)$. Then $f_{2}\left(z_{0}\right)=P\left(z_{0}\right) y\left(z_{0}\right)+Q\left(z_{0}\right)=0$. Thus, $y\left(z_{0}\right)=$ $-Q\left(z_{0}\right) / P\left(z_{0}\right)$. Substituting $y\left(z_{0}\right)=-Q\left(z_{0}\right) / P\left(z_{0}\right)$ into $f_{1}(z)$, we obtain

$$
\begin{aligned}
f_{1}\left(z_{0}\right) & =-\frac{Q\left(z_{0}\right)}{P\left(z_{0}\right)}\left(R\left(z_{0}\right)-z_{0} P\left(z_{0}\right)\right)-z_{0} Q\left(z_{0}\right) \\
& =-\frac{R\left(z_{0}\right) Q\left(z_{0}\right)}{P\left(z_{0}\right)} \\
& =0 .
\end{aligned}
$$

Since $R(z) Q(z) / P(z)$ has only finitely many zeros, we see that $f_{1}(z)$ and $f_{2}(z)$ have at most finitely many common zeros. 
Lemma 7 (see [14]). Let $f(z)$ be a nonconstant finite order meromorphic function. Then

$$
T(r+1, f(z))=T(r, f(z))+S(r, f(z)) .
$$

Gol'dberg and Ostrovskii [21, page 66] give that for any constant $b$,

$$
\begin{aligned}
& (1+o(1)) T(r-|b|, f(z)) \\
& \quad \leq T(r, f(z+b)) \leq(1+o(1)) T(r+|b|, f(z)) .
\end{aligned}
$$

This and Lemma 7 give the following lemma.

Lemma 8. Let $f(z)$ be a nonconstant finite order meromorphic function. Then

$$
T(r, f(z+1))=T(r, f(z))+S(r, f(z)) .
$$

Using the same method as in the proof of Lemma 6, we can prove Lemmas 9 and 10 .

Lemma 9. Suppose that $R(z), Q(z)$, and $P(z)$ satisfy the condition (8) in Theorem 2 and that $y(z)$ is a nonconstant meromorphic function. Then

$$
\begin{gathered}
f_{1}(z)=R(z)-(z+1) Q(z)-(z+1) P(z) y(z), \\
f_{2}(z)=P(z) y(z)+Q(z)
\end{gathered}
$$

have at most finitely many common zeros.

Lemma 10. Suppose that $R(z), Q(z)$, and $P(z)$ satisfy the condition (8) in Theorem 2 and $y(z)$ is a nonconstant meromorphic function. Then

$$
\begin{gathered}
f_{1}(z)=P(z) y(z)^{2}+[-R(z)+Q(z)+z P(z)] y(z) \\
+z Q(z), \\
f_{2}(z)=P(z) y(z)+Q(z)
\end{gathered}
$$

have at most finitely many common zeros.

Proof of Theorem 2. (i) We prove that $\tau(y(z+n))=\sigma(y(z)) \geq$ $1(n=0,1, \ldots)$. Suppose that $n=0$. Set $y(z)-z=g(z)$. So, $g(z)$ is transcendental, $T(r, g(z))=T(r, y(z))+O(\log r)$, and $S(r, g)=S(r, y)$. Substituting $y(z)=g(z)+z$ into (2), we obtain

$$
\begin{aligned}
K_{0}(z, g):= & P(z)[g(z+1)+z+1][g(z)+z] \\
& +Q(z)[g(z+1)+z+1] \\
& -R(z)[g(z)+z]=0 .
\end{aligned}
$$

Thus,

$$
K_{0}(z, 0)=z(z+1) P(z)+(z+1) Q(z)-z R(z) .
$$

By (8) and (22), we see that $K_{0}(z, 0) \not \equiv 0$. Thus, by Lemma 4 and $K_{0}(z, 0) \not \equiv 0$, we obtain

$$
N\left(r, \frac{1}{g(z)}\right)=T(r, g(z))+S(r, g(z))
$$

for all $r$ outside of a possible exceptional set with finite logarithmic measure. Thus,

$$
N\left(r, \frac{1}{y(z)-z}\right)=T(r, y(z))+S(r, y(z))
$$

for all $r$ outside of a possible exceptional set with finite logarithmic measure. So, by Theorem A and (24), we obtain $\tau(y(z))=\sigma(y(z)) \geq 1$.

Now suppose that $n=1$. By (2), we obtain

$$
\begin{aligned}
y(z+1)-z & \\
= & \frac{(R(z)-z P(z)) y(z)-z Q(z)}{Q(z)+P(z) y(z)} \\
= & \frac{(R(z)-z P(z))[y(z)-z Q(z) /(R(z)-z P(z))]}{Q(z)+P(z) y(z)} .
\end{aligned}
$$

By (8), we see that $R(z)-z P(z) \not \equiv 0$. Since $P(z), Q(z)$, and $R(z)$ are polynomials, by $(25)$, we see that $y(z)-z Q(z) /(R(z)-$ $z P(z))$ and $Q(z)+P(z) y(z)$ have the same poles, except possibly finitely many poles. By Lemma 6 , we see that $(R(z)-$ $z P(z)) y(z)-z Q(z)$ and $Q(z)+P(z) y(z)$ have at most finitely many common zeros. Hence, by (25), we have that

$$
\begin{aligned}
\tau(y(z+1)) & =\lambda(y(z+1)-z) \\
& =\lambda\left(y(z)-\frac{z Q(z)}{R(z)-z P(z)}\right) .
\end{aligned}
$$

Suppose that $\lambda(y(z)-z Q(z) /(R(z)-z P(z)))<\sigma(y(z))$. Thus, $y(z)-z Q(z) /(R(z)-z P(z))$ can be rewritten as the following form:

$$
y(z)-\frac{z Q(z)}{R(z)-z P(z)}=z^{s} \frac{b_{0}(z)}{H_{0}(z)} e^{h(z)}=\frac{b(z)}{H(z)},
$$

where $h(z)$ is a polynomial with $\operatorname{deg} h(z) \leq \sigma(y(z)), b_{0}(z)$ and $H_{0}(z)$ are canonical products $\left(b_{0}(z)\right.$ may be a polynomial) formed by nonzero zeros and poles of $y(z)-$ $z Q(z) /(R(z)-z P(z))$, respectively, and $s$ is an integer; if $s \geq 0$, then $b(z)=z^{s} b_{0}(z), H(z)=H_{0}(z) e^{-h(z)}$; if $s<0$, then $b(z)=b_{0}(z), H(z)=z^{-s} H_{0}(z) e^{-h(z)}$. Combining Theorem A with properties of canonical product, we see that

$$
\begin{aligned}
\lambda(b(z)) & =\sigma(b(z)) \\
& =\lambda\left(y(z)-\frac{z Q(z)}{R(z)-z P(z)}\right) \\
< & \sigma(y(z)), \\
\lambda(H(z)) & =\sigma(H(z))=\sigma(y(z)) .
\end{aligned}
$$

By (27), we obtain

$$
\begin{gathered}
y(z)=\frac{z Q(z)}{R(z)-z P(z)}+b(z) f(z), \\
y(z+1)=\frac{(z+1) Q(z+1)}{R(z+1)-(z+1) P(z+1)} \\
+b(z+1) f(z+1),
\end{gathered}
$$


where $f(z)=1 / H(z)$. Thus, by (28) and Lemma 8 , we have that

$$
\begin{gathered}
\sigma(f(z))=\sigma(H(z))=\sigma(y(z)), \\
\sigma(b(z+1))=\sigma(b(z))<\sigma(f(z)) .
\end{gathered}
$$

Substituting (29) into (2), we obtain

$$
\begin{aligned}
K_{1} & (z, f) \\
:= & \left\{\frac{(z+1) Q(z+1)}{R(z+1)-(z+1) P(z+1)}+b(z+1) f(z+1)\right\} \\
& \cdot\left\{Q(z)+P(z)\left[\frac{z Q(z)}{R(z)-z P(z)}+b(z) f(z)\right]\right\} \\
& -R(z)\left[\frac{z Q(z)}{R(z)-z P(z)}+b(z) f(z)\right]=0 .
\end{aligned}
$$

By (31), we obtain

$$
\begin{aligned}
& K_{1}(z, 0) \\
& =[(z+1) Q(z+1) Q(z) R(z) \\
& \quad-z Q(z) R(z)[R(z+1)-(z+1) P(z+1)]] \\
& \quad \times([R(z+1)-(z+1) P(z+1)][R(z)-z P(z)])^{-1} .
\end{aligned}
$$

By (8), we see that in the numerator of the right side of (32), there exists only one term $z(z+1) Q(z) R(z) P(z+1)$ being of the highest degree. So,

$$
K_{1}(z, 0) \not \equiv 0 .
$$

Thus, by (28), (33), Lemma 4, and its Remark 5, we obtain that for any given $\varepsilon(0<\varepsilon<\sigma(y(z))-\sigma(b(z)))$

$$
\begin{aligned}
N\left(r, \frac{1}{f(z)}\right)= & T(r, f(z))+S(r, f(z)) \\
& +O\left(r^{\sigma(b(z))+\varepsilon}\right)
\end{aligned}
$$

holds for all $r$ outside of a possible exceptional set with finite logarithmic measure.

On the other hand, by $f(z)=1 / H(z)$ and the fact that $H(z)$ is an entire function, we see that

$$
N\left(r, \frac{1}{f(z)}\right)=0 .
$$

Thus, by this and (28), we see that (34) is a contradiction. Hence, $\lambda(y(z)-z Q(z) /(R(z)-z P(z)))=\sigma(y(z))$. By (26), we obtain

$$
\tau(y(z+1))=\sigma(y(z)) .
$$

Now suppose that $n=2$. By (2), we obtain

$$
g(z+1)=\frac{R(z+1) g(z)}{Q(z+1)+P(z+1) g(z)},
$$

where $g(z)=y(z+1)$. By Lemma 8 , we have that $\sigma(g(z))=$ $\sigma(y(z))$. By (8), we have

$$
\begin{array}{r}
\operatorname{deg} P(z+1) \geq \max \{\operatorname{deg} R(z+1), \operatorname{deg} Q(z+1)\}, \\
\operatorname{deg} P(z+1) \geq 1 .
\end{array}
$$

Thus, for (37), applying the conclusion of $n=1$ above, we obtain

$$
\tau(y(z+2))=\tau(g(z+1))=\sigma(g(z))=\sigma(y(z)) .
$$

Continuing to use the same method as above, we can obtain

$$
\tau(y(z+n))=\sigma(y(z)) \quad(n=1,2, \ldots) .
$$

(ii) Suppose that $R(z)-(z+1) Q(z) \neq \equiv 0$. We prove that $\tau(\Delta y(z) / y(z))=\sigma(y(z))$. By (2), we obtain

$$
\begin{aligned}
& \frac{\Delta y(z)}{y(z)}-z \\
& =\frac{y(z+1)-y(z)}{y(z)}-z \\
& =\frac{R(z)-(z+1) Q(z)-(z+1) P(z) y(z)}{Q(z)+P(z) y(z)} \\
& =-(z+1) P(z) \\
& \quad \times(y(z)-(R(z)-(z+1) Q(z)) /(z+1) P(z)) \\
& \quad \times(Q(z)+P(z) y(z))^{-1} .
\end{aligned}
$$

Since $y(z)-(R(z)-(z+1) Q(z)) /(z+1) P(z)$ and $Q(z)+$ $P(z) y(z)$ have the same poles, except possibly finitely many poles, by Lemma 9 and (41), we only need to prove that

$$
\lambda\left(y(z)-\frac{R(z)-(z+1) Q(z)}{(z+1) P(z)}\right)=\sigma(y(z)) .
$$

Set

$$
h_{1}(z)=R(z)-(z+1) Q(z), \quad h_{2}(z)=(z+1) P(z) .
$$

Suppose that $\lambda\left(y(z)-h_{1}(z) / h_{2}(z)\right)<\sigma(y(z))$. Using the same method as in the proof of (i), $y(z)-h_{1}(z) / h_{2}(z)$ can be rewritten as the following form:

$$
y(z)=\frac{h_{1}(z)}{h_{2}(z)}+b_{2}(z) f_{2}(z)
$$

where $f_{2}(z)=1 / H_{2}(z)$ and $b_{2}(z)$ and $H_{2}(z)$ are nonzero entire functions, such that

$$
\begin{aligned}
\lambda\left(b_{2}(z)\right) & =\sigma\left(b_{2}(z)\right)<\sigma(y(z)), \\
\lambda\left(H_{2}(z)\right) & =\sigma\left(H_{2}(z)\right)=\sigma(y(z)) .
\end{aligned}
$$


Substituting (44) into (2), we obtain

$$
\begin{aligned}
D_{2} & \left(z, f_{2}(z)\right) \\
:= & \left\{\frac{h_{1}(z+1)}{h_{2}(z+1)}+b_{2}(z+1) f_{2}(z+1)\right\} \\
& \times\left\{Q(z)+P(z) \frac{h_{1}(z)}{h_{2}(z)}+P(z) b_{2}(z) f_{2}(z)\right\} \\
& -R(z)\left\{\frac{h_{1}(z)}{h_{2}(z)}+b_{2}(z) f_{2}(z)\right\}=0, \\
D_{2} & (z, 0) \\
= & \frac{h_{1}(z+1)}{h_{2}(z+1)}\left\{Q(z)+P(z) \frac{h_{1}(z)}{h_{2}(z)}\right\}-R(z) \frac{h_{1}(z)}{h_{2}(z)} \\
= & \frac{h_{1}(z+1)}{h_{2}(z+1)}\left\{Q(z)+P(z) \frac{R(z)-(z+1) Q(z)}{(z+1) P(z)}\right\} \\
& -R(z) \frac{h_{1}(z)}{h_{2}(z)} \\
= & R(z) \frac{h_{1}(z+1) h_{2}(z)-(z+1) h_{1}(z) h_{2}(z+1)}{(z+1) h_{2}(z+1) h_{2}(z)} .
\end{aligned}
$$

Since $h_{1}(z)$ and $h_{2}(z)$ are polynomials, we see that

$$
h_{1}(z+1) h_{2}(z)-(z+1) h_{1}(z) h_{2}(z+1) \not \equiv 0,
$$

that is,

$$
D_{2}(z, 0) \not \equiv 0 .
$$

Using the same method as in the proof of (i), we obtain a contradiction. Hence, (42) holds; that is, $\tau(\Delta y(z) / y(z))=$ $\sigma(y(z))$.

(iii) Suppose that there is a polynomial $h(z)$ satisfying

$$
(-R(z)+Q(z)+z P(z))^{2}-4 z P(z) Q(z)=h(z)^{2} .
$$

Thus, by (8) and (49), we see that

$$
\operatorname{deg} h(z)=\operatorname{deg} P(z)+1 .
$$

Now we prove that $\tau(\Delta y(z))=\sigma(y(z))$. By (2), we obtain

$$
\begin{aligned}
& y(z+1)-y(z)-z \\
& =-\frac{P(z) y(z)^{2}+[-R(z)+Q(z)+z P(z)] y(z)+z Q(z)}{Q(z)+P(z) y(z)} .
\end{aligned}
$$

By (49) and (51), we obtain

$$
\begin{aligned}
& y(z+1)-y(z)-z \\
& =-P(z)\left\{y(z)-\frac{-(z P(z)+Q(z)-R(z))+h(z)}{2 P(z)}\right\} \\
& \quad \times\left\{y(z)-\frac{-(z P(z)+Q(z)-R(z))-h(z)}{2 P(z)}\right\} \\
& \quad \times(Q(z)+P(z) y(z))^{-1} .
\end{aligned}
$$

Since $P(z), Q(z)$, and $R(z)$ are polynomials, we see that poles of $Q(z)+P(z) y(z)$ must be poles of $P(z) y(z)^{2}+[-R(z)+$ $Q(z)+z P(z)] y(z)+z Q(z)$. Thus, poles of $Q(z)+P(z) y(z)$ are not zeros of $y(z+1)-y(z)-z$. By Lemma 10, we see that the numerator and the denominator of the right side of (51) have at most finitely many common zeros. Thus, in order to prove $\tau(\Delta y(z))=\sigma(y(z))$, by (52), we only need to prove that

$$
\lambda\left(y(z)-\frac{-(z P(z)+Q(z)-R(z))+h(z)}{2 P(z)}\right)=\sigma(y(z))
$$

or

$$
\lambda\left(y(z)-\frac{-(z P(z)+Q(z)-R(z))-h(z)}{2 P(z)}\right)=\sigma(y(z)) .
$$

By (50), we have $\operatorname{deg}(-z P(z))=\operatorname{deg} h(z)=\operatorname{deg} P(z)+1$. Combining this with (8), we see that there exists at least one of

$$
\begin{aligned}
& -(z P(z)+Q(z)-R(z))+h(z), \\
& -(z P(z)+Q(z)-R(z))-h(z),
\end{aligned}
$$

such that its degree is equal to $\operatorname{deg} P(z)+1$. Without loss of generality, we may suppose that

$$
\operatorname{deg}(-(z P(z)+Q(z)-R(z))+h(z))=\operatorname{deg} P(z)+1 .
$$

Now we prove that (53) holds. Suppose that

$$
\lambda\left(y(z)-\frac{-(z P(z)+Q(z)-R(z))+h(z)}{2 P(z)}\right)<\sigma(y(z)) .
$$

Using a similar method as in the proof of (i), we see that $y(z)-$ $(-(z P(z)+Q(z)-R(z))+h(z)) / 2 P(z)$ can be rewritten as the following form:

$$
\begin{aligned}
y(z)= & \frac{-(z P(z)+Q(z)-R(z))+h(z)}{2 P(z)} \\
& +b_{3}(z) f_{3}(z),
\end{aligned}
$$

where $f_{3}(z)=1 / H_{3}(z)$ and $b_{3}(z)$ and $H_{3}(z)$ are nonzero entire functions, such that

$$
\begin{gathered}
\lambda\left(b_{3}(z)\right)=\sigma\left(b_{3}(z)\right)<\sigma(y(z)), \\
\lambda\left(H_{3}(z)\right)=\sigma\left(H_{3}(z)\right)=\sigma(y(z)) .
\end{gathered}
$$


Substituting (58) into (2), we obtain

$$
\begin{aligned}
& D_{3}\left(z, f_{3}(z)\right) \\
& :=\left\{\frac{-((z+1) P(z+1)+Q(z+1)-R(z+1))+h(z+1)}{2 P(z+1)}\right. \\
& \left.+b_{3}(z+1) f_{3}(z+1)\right\} \\
& \cdot\left\{Q(z)+P(z) \frac{-(z P(z)+Q(z)-R(z))+h(z)}{2 P(z)}\right. \\
& \left.+P(z) b_{3}(z) f_{3}(z)\right\} \\
& -R(z) \frac{-(z P(z)+Q(z)-R(z))+h(z)}{2 P(z)} \\
& -R(z) b_{3}(z) f_{3}(z)=0, \\
& D_{3}(z, 0) \\
& :=\left\{\frac{-(z+1) P(z+1)-Q(z+1)+R(z+1)+h(z+1)}{2 P(z+1)}\right\} \\
& \left\{\frac{-z P(z)+Q(z)+R(z)+h(z)}{2}\right\} \\
& -R(z) \frac{-z P(z)-Q(z)+R(z)+h(z)}{2 P(z)} \\
& =\frac{W_{1}(z)-W_{2}(z)}{4 P(z+1) P(z)},
\end{aligned}
$$

where

$$
\begin{gathered}
W_{1}(z)=[-(z+1) P(z+1)-Q(z+1) \\
+R(z+1)+h(z+1)] \\
\times[-z P(z)+Q(z)+R(z)+h(z)] P(z), \\
W_{2}(z)=2(-z P(z)-Q(z)+R(z)+h(z)) R(z) P(z+1) .
\end{gathered}
$$

By (8), (50), and (56), we see that

$$
\begin{gathered}
\operatorname{deg}(-(z+1) P(z+1)-Q(z+1)+R(z+1)+h(z+1)) \\
=\operatorname{deg} P(z)+1, \\
\operatorname{deg}(-z P(z)+Q(z)+R(z)+h(z))=\operatorname{deg} P(z)+1 .
\end{gathered}
$$

Thus, we obtain

$$
\begin{aligned}
& \operatorname{deg} W_{1}(z)=3 \operatorname{deg} P(z)+2, \\
& \operatorname{deg} W_{2}(z) \leq 3 \operatorname{deg} P(z)+1
\end{aligned}
$$

So, by $(60)$ and (63), we see that $D_{3}(z, 0) \not \equiv 0$.

Using the same method as in the proof of (i), we see that (53) holds.

Thus, Theorem 2 is proved.

\section{Acknowledgments}

The first author was supported by the National Natural Science Foundation of China (no. 11171119). The second author was supported by Basic Science Research Program through the National Research Foundation of Korea (NRF) funded by the Ministry of Education, Science and Technology (2010-0009646).

\section{References}

[1] W. K. Hayman, Meromorphic Functions, Oxford Mathematical Monographs, Clarendon Press, Oxford, UK, 1964.

[2] I. Laine, Nevanlinna Theory and Complex Differential Equations, vol. 15 of de Gruyter Studies in Mathematics, Walter de Gruyter, Berlin, Germany, 1993.

[3] L. Yang, Value Distribution Theory, Science Press, Beijing, China, 1993.

[4] M. J. Ablowitz, R. Halburd, and B. Herbst, "On the extension of the Painlevé property to difference equations," Nonlinearity, vol. 13, no. 3, pp. 889-905, 2000.

[5] W. Bergweiler and J. K. Langley, "Zeros of differences of meromorphic functions," Mathematical Proceedings of the Cambridge Philosophical Society, vol. 142, no. 1, pp. 133-147, 2007.

[6] B. Q. Chen, Z. X. Chen, and S. Li, "Uniqueness theorems on entire functions and their difference operators or shifts," Abstract and Applied Analysis, vol. 2012, Article ID 906893, 8 pages, 2012.

[7] Z. Chen, "On growth, zeros and poles of meromorphic solutions of linear and nonlinear difference equations," Science China. Mathematics, vol. 54, no. 10, pp. 2123-2133, 2011.

[8] Z.-X. Chen and K. H. Shon, "On zeros and fixed points of differences of meromorphic functions," Journal of Mathematical Analysis and Applications, vol. 344, no. 1, pp. 373-383, 2008.

[9] Z.-X. Chen and K. H. Shon, "Properties of differences of meromorphic functions," Czechoslovak Mathematical Journal, vol. 61, no. 1, pp. 213-224, 2011.

[10] Y.-M. Chiang and S.-J. Feng, "On the Nevanlinna characteristic of $f(z+\eta)$ and difference equations in the complex plane," Ramanujan Journal, vol. 16, no. 1, pp. 105-129, 2008.

[11] Y.-M. Chiang and S.-J. Feng, "On the growth of logarithmic differences, difference quotients and logarithmic derivatives of meromorphic functions," Transactions of the American Mathematical Society, vol. 361, no. 7, pp. 3767-3791, 2009.

[12] R. G. Halburd and R. J. Korhonen, "Difference analogue of the lemma on the logarithmic derivative with applications to difference equations," Journal of Mathematical Analysis and Applications, vol. 314, no. 2, pp. 477-487, 2006.

[13] R. G. Halburd and R. J. Korhonen, "Existence of finite-order meromorphic solutions as a detector of integrability in difference equations," Physica D, vol. 218, no. 2, pp. 191-203, 2006.

[14] R. G. Halburd and R. J. Korhonen, "Meromorphic solutions of difference equations, integrability and the discrete Painlevé equations," Journal of Physics A, vol. 40, no. 6, pp. R1-R38, 2007.

[15] J. Heittokangas, R. Korhonen, I. Laine, J. Rieppo, and J. Zhang, "Value sharing results for shifts of meromorphic functions, and sufficient conditions for periodicity," Journal of Mathematical Analysis and Applications, vol. 355, no. 1, pp. 352-363, 2009.

[16] J. Heittokangas, R. Korhonen, I. Laine, J. Rieppo, and K. Tohge, "Complex difference equations of Malmquist type," 
Computational Methods and Function Theory, vol. 1, no. 1, pp. 27-39, 2001.

[17] I. Laine and C.-C. Yang, "Clunie theorems for difference and q-difference polynomials," Journal of the London Mathematical Society. Second Series, vol. 76, no. 3, pp. 556-566, 2007.

[18] S. Elaydi, An Introduction to Difference Equations, Undergraduate Texts in Mathematics, Springer, New York, NY, USA, 3rd edition, 2005.

[19] W. Bergweiler, "Proof of a conjecture of Gross concerning fixpoints," Mathematische Zeitschrift, vol. 204, no. 3, pp. 381-390, 1990.

[20] C.-T. Chuang and C.-C. Yang, Fix-Points and Factorization of Meromorphic Functions, World Scientific, Singapore, 1990.

[21] A. A. Gol'dberg and I. V. Ostrovskiĭ, The Distribution of Values of Meromorphic Functions, Nauka, Moscow, Russia, 1970. 


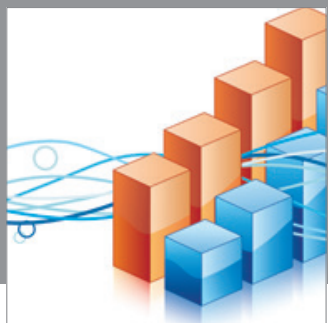

Advances in

Operations Research

mansans

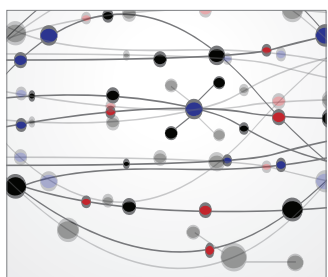

The Scientific World Journal
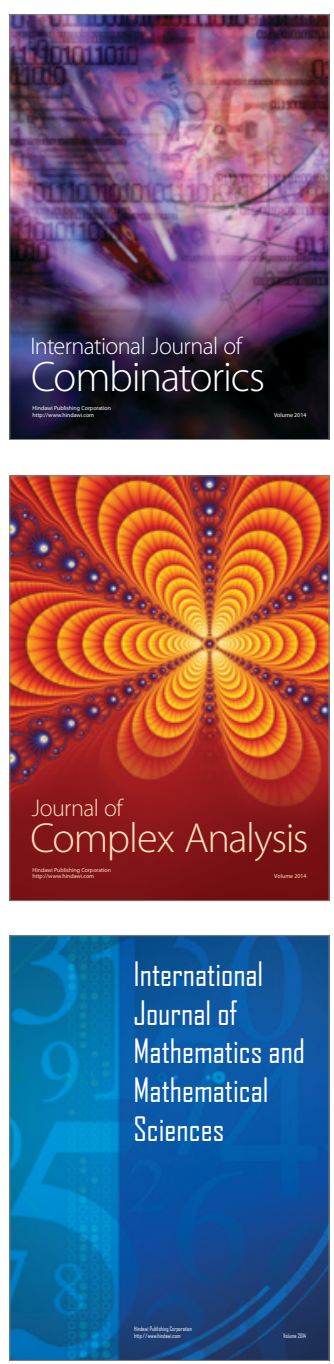
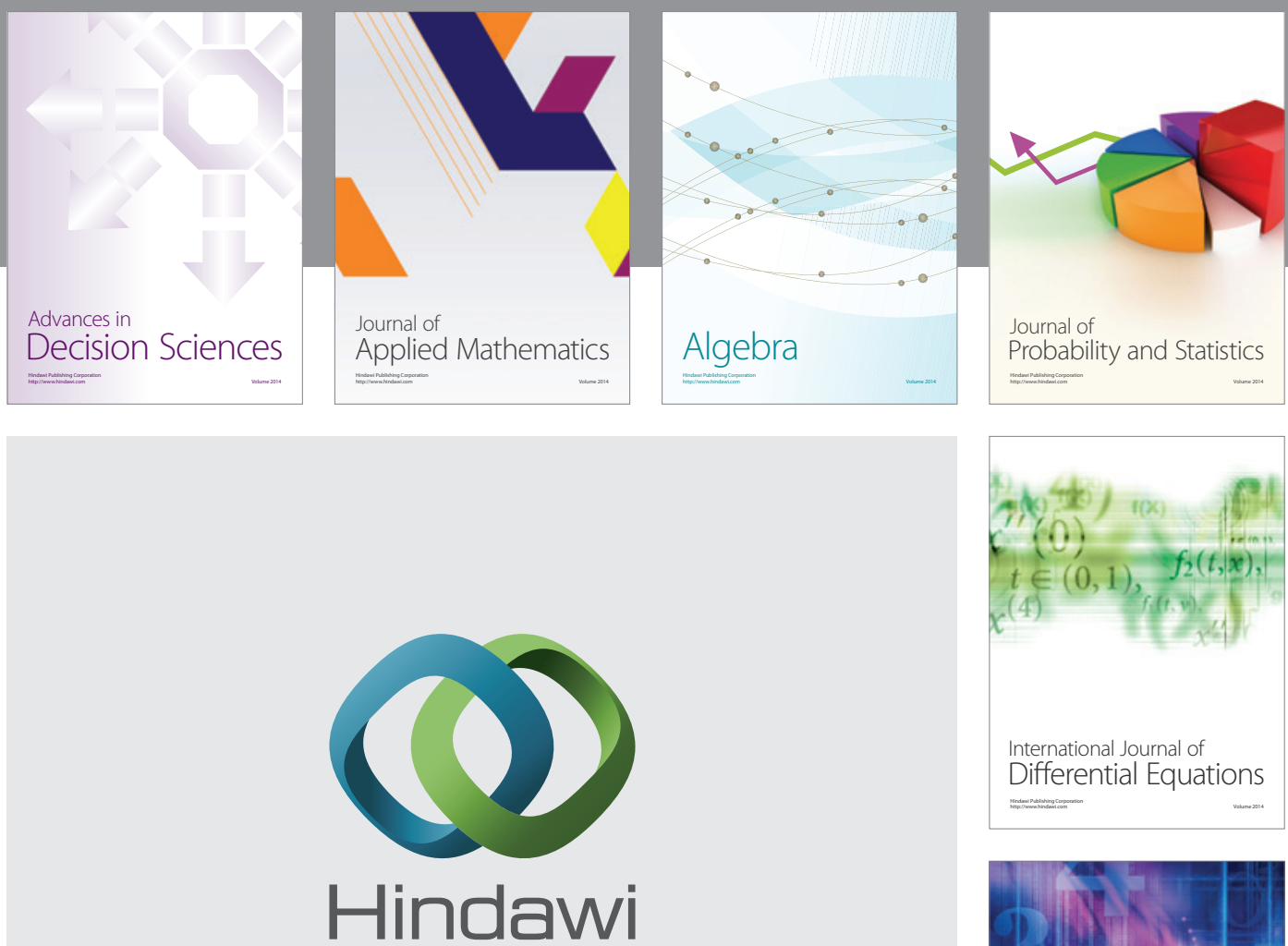

Submit your manuscripts at http://www.hindawi.com
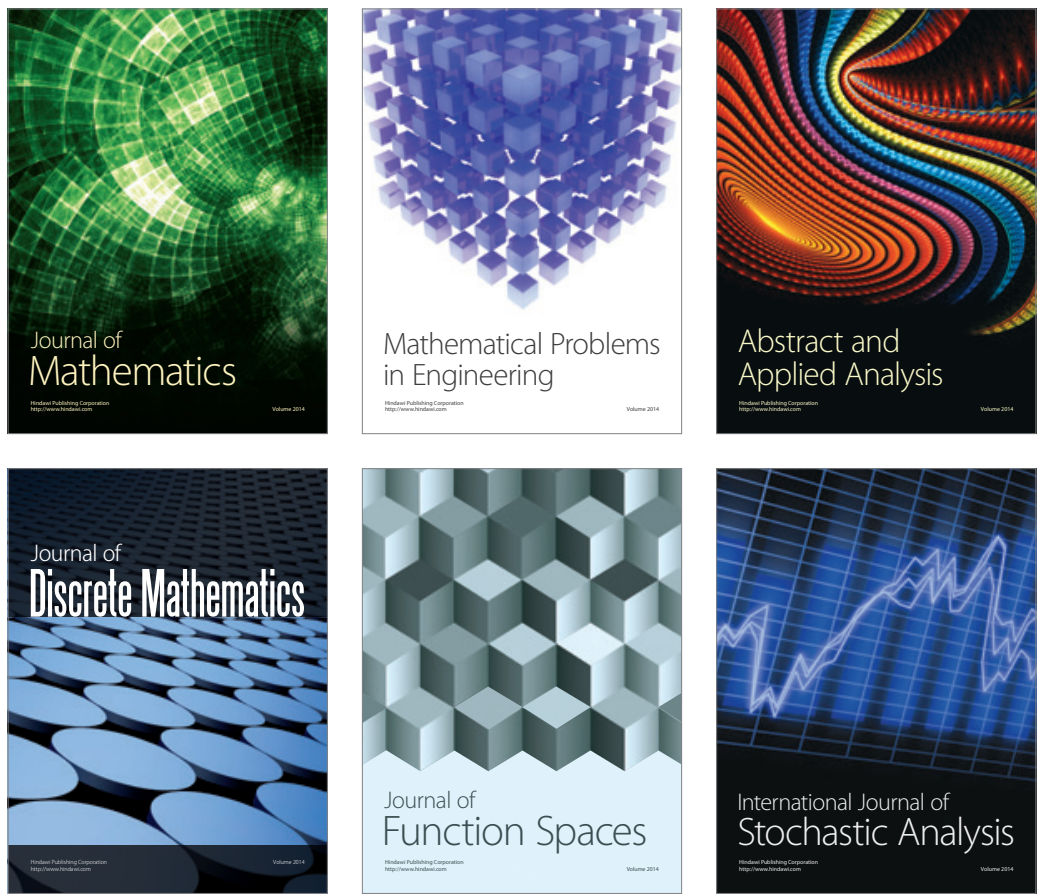

Journal of

Function Spaces

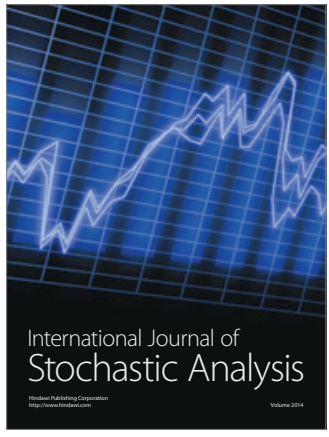

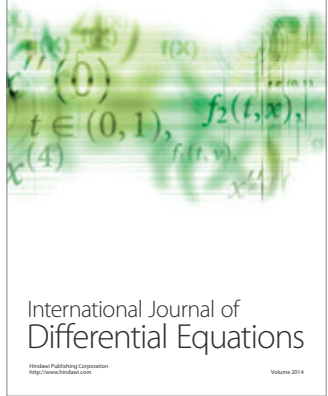
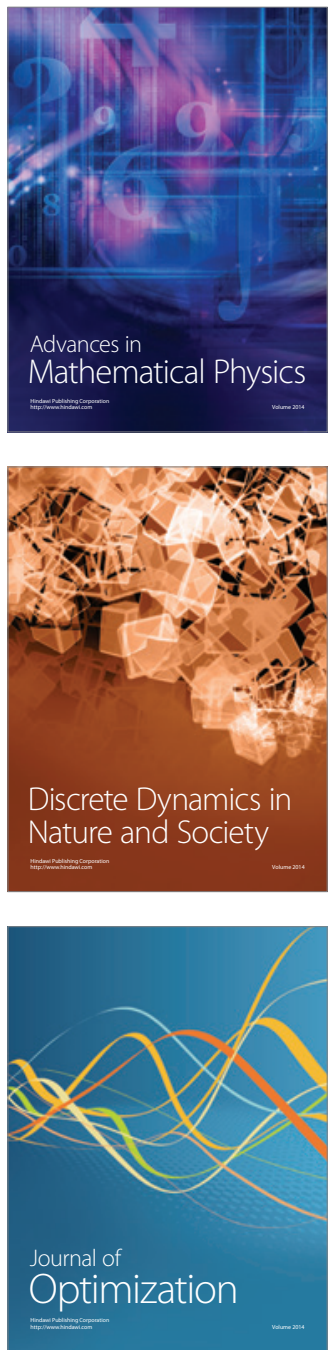